\title{
Chronic heart failure in the elderly: a current medical problem
}

\author{
Jadwiga Nessler, Agnieszka Skrzypek \\ Department of Coronary Heart Disease, Institute of Cardiology, Jagiellonian University School of Medicine, John Paul II Hospital, Kraków, Poland
}

\section{KEY WORDS}

elderly, heart failure, pathophysiology, therapy
Correspondence to:

Jadwiga Nessler, MD, PhD, Klinika Choroby Wieńcowej, Instytut Kardiologii, Uniwersytet Jagielloński, Collegium Medicum, Krakowski Szpital Specjalistyczny im. Jana Pawła II, ul. Prądnicka 80, 31-202 Kraków, Poland,

phone/fax: +48-12-633-67-44 e-mail: jnessler@interia.pl Received: January 20, 2008 Revision accepted: May 22, 2008. Conflict of interest: none declared. Pol Arch Med Wewn. 2008; 118 (10): 572-580

Translated by Professional Language Services SIGILLUM Ltd., Kraków Copyright by Medycyna Praktyczna, Kraków 2008

\section{ABSTRACT}

As a result of population ageing and improved medical care that contribute to better life expectancy, heart failure occurs more and more commonly in the elderly. In the USA approximately $80 \%$ of patients discharged from hospital with newly diagnosed heart failure are over 65 years of age, whereas $50 \%$ are over 75 . The average 5 -year mortality rate is about $50 \%$ in subjects with systolic dysfunction and similar in those with preserved left ventricular systolic function. Disorders of the cardiovascular system occurring in the elderly (e.g. increased left ventricular mass, myocardial rigidity, atrial fibrillation, decreased maximum oxygen uptake in cardiopulmonary exercise tests) result from the physiological ageing; they may also be caused by a concomitant cardiac failure syndrome. In the elderly, heart failure is often accompanied by concomitant conditions that often make diagnosis and treatment of chronic heart disease difficult. Non-specific clinical symptoms in the elderly as well as those associated with age (e.g. easy fatigability, exertional dyspnea) make a correct diagnosis difficult. The recognized biochemical marker of heart failure - brain natriuretic peptide, $\mathrm{N}$-terminal pro-brain natriuretic peptide - has a limited diagnostic value in the elderly. Echocardiography plays a key role in the diagnosis. Owing to altered metabolism, impairment of hepatic processes to various degrees and decreased renal excretion of drugs, treatment requires attention, individual choice of drugs and doses, as well as periodic modification of both the doses and the intervals between them. Correct treatment improves quality of life and prolongs it. The aim of the present work is to present the differences in the pathophysiology, diagnostic evaluation and management of chronic heart failure in the elderly, in light of the current views and standards.

INTRODUCTION Due to its prognosis, chronic heart failure (CHF) is a grave stage of many conditions. About $2 \%$ of the American population and about $1.8 \%$ of Europeans are treated for heart failure. ${ }^{1,2}$ Owing to population ageing and improvements in the health care system that contribute to the prolongation of life, there is an increasing number of the elderly with CHF. According to the Cardiovascular Health Study, in the population aged $66-103(n=4842)$, symptomatic heart failure occurred at the rate of 19.3 for 1000 person-years. The rate rose from 10.6 for 1000 person-years in patients aged 65-69 in the initial study, to 42.5 for 1000 person-years in patients aged $>80 .{ }^{3}$ Asymptomatic systolic left ventricular dysfunction in the older age groups occurs in additional $3-5 \%$ of individuals. ${ }^{4}$
Heart failure is currently one of the major causes of hospitalization in the USA and is responsible for almost a million hospital admissions yearly. About $80 \%$ of patients discharged from hospital with newly recognised heart failure are $>65$ years old, whereas $50 \%$ are $>75 .{ }^{5}$ Despite the advances in treatment, the average 5-year mortality rate is approximately $50 \%$ in subjects with systolic dysfunction and similar in those with preserved left ventricular ejection fraction. ${ }^{6}$ The average survival duration from the diagnosis of heart failure varies between sexes; it is 1.7 years in men and 3.2 years in women. ${ }^{7}$ The DIAMOND study indicated that among patients hospitalized for $\mathrm{CHF}$ the male sex was an independent risk factor of death. ${ }^{8}$

In elderly persons, heart failure is often accompanied by other concomitant conditions 
FIGURE Pathophysiological similarities between the elderly and failing heart

$\uparrow$-increase,

$\downarrow$-decrease

Abbreviations:

LV - left ventricular



and changes resulting from the ageing process. That is why diagnosing $\mathrm{CHF}$ in old age is more difficult. Owing to the altered metabolism, impairment of the hepatic processes to various degrees and decreased renal excretion of drugs, treatment requires attention, individual choice of drugs and their periodic modification. Appropriate treatment improves and prolongs life. The aim of the present work is to present differences in the pathophysiology, diagnostic evaluation and management of CHF in the elderly, taking into consideration the current views and standards.

Definition of senility There is no universal definition of senility. Physiological changes occurring with the passage of years do not appear at any definite time; nor are they equally marked in every person. The majority of definitions of senility are based on calendar age. The World Health Organisation defines senility as the age of $>60$, whereas according to most American classifications the borderline between the maturity and senility is 65 years. Gerontologists distinguish between 3 subgroups: younger older people (60-74 years), older older people (75-85 years) and very old people (> 85 years). Due to the fact that considerable decreases in physical and mental efficiency, as well as multi-organ disorders occur much more frequently beyond the age of 80 , clinicians distinguish between 2 subgroups of older patients: i.e. those under and those above that age. ${ }^{9}$
Age-related changes in the cardiovascular system In elderly people disorders occur that are closely related to the ageing process. They include symptoms from the cardiovascular system, such as elevated systolic blood pressure, pulse pressure (the difference between the systolic and diastolic arterial pressures), increased mass of the left ventricle and more frequent ischemic heart disease, as well as cardiac arrhythmias, and particularly atrial fibrillation. With age there is a decrease in the ability to increase cardiac output in response to stress. In comparison with young people, with physical exertion the maximum values of such parameters as early diastolic filling pressure, maximum heart rate, maximum cardiac output, maximum oxygen consumption, exertion-induced increase in ejection fraction are achieved sooner. Increased heart rate and vascular dilation in response to $\beta$-adrenergic come sooner, too. ${ }^{10-11}$

This results in the loss of elastic fibers and enhanced fibrosis and calcification. In blood vessels the lumen becomes broader and the intima becomes thicker, which leads to increased rigidity of the vessels and the elevation of the pulse wave in the peripheral blood vessels. The decreased production of nitrogen oxide by endothelial cells is found in both human and animal models. This leads to decreased vasodilatatory activity of the coronary and peripheral vessels. ${ }^{12}$

Over years vascular response to $\beta$-agonists and $\beta$-adrenergic inhibitors is reduced, too. In contrast, response to vasodilators (e.g. nitrates, stimulation or endothelium-released vasodilators 
nitroprusside) is not altered with age, but may be impaired by concomitant arterial hypertension or diabetes mellitus. Along with the vascular abnormalities there are also alterations in the extracellular matrix of the myocardium such as increased collagen content, greater diameter of myofibrils, decreased elastin content and elevated fibronectin levels. These changes are accompanied by the decrease in the number of cells as a result of apoptosis (i.e. programmed cell death) and the impairment of their function. ${ }^{9}$ Other changes associated with age include the presence of collagen deposits in the ventricles and altered extracellular matrix leading to hypertrophy and decrease in the number of myocytes, as well as disorders in the myosin structure. Disorders of calcium uptake ensue resulting in longer systole and diastole. Deposits of amyloid and lipofuscin cause the loss of elasticity and compliance of the myocardium, which is characteristic of the senile heart. ${ }^{13}$ Regardless of etiology (age, coronary artery disease or arterial hypertension) impaired of myocardial compliance is considered to be the major cause of diastolic heart failure in older people. ${ }^{13-15}$

Diastolic CHF mainly affects older patients with concomitant arterial hypertension and left ventricular hypertrophy. It results from myocardial rigidity secondary to extracellular matrix accumulation and the formation of cross junctions between structural protein fibres. ${ }^{16}$ In the ageing heart, the cross junctions between collagen fibres predispose to macrophage accumulation in the myocardium, thus causing its fibrosis and rigidity. This leads to the deterioration of cardiac diastolic function. ${ }^{17}$

A decrease in the number of sinus node cells in the atrium as a result of fibrosis and changes of the composition of extracellular matrix lead to the sinus node dysfunction and contribute to atrial fibrillation, which considerably deteriorates the end-diastolic left ventricular filling. ${ }^{18}$ An increased amount of collagen, decreased amount of elastic tissue, as well as calcification in the vicinity of the atrioventricular node and the His bundle branches are responsible for the conduction disturbances and the calcification of the valvular annuli. ${ }^{9}$

Age-related changes are also present in the circulating blood. The levels of fibrinogen, factors V, VIII, IX and other coagulation proteins are elevated without the concomitant increase in the concentration of coagulation inhibitors. The concentration of phospholipids in platelets is elevated, which results in enhanced platelet aggregability. Plasminogen activator inhibitor-1 (PAI-1) activity is higher, with the resultant decrease in intravascular fibrinolysis. Elevated levels of pro-inflammatory cytokines with pro-coagulant activity predominantly of interleukin- 6 are observed, which may play a role in a higher prevalence of acute coronary syndromes. The source of PAI- 1 and pro-inflammatory cytokines are adipocytes. ${ }^{12,19}$ The above-mentioned neurohormonal changes accelerate the development of atherosclerosis.
With age, changes in the regulation of the autonomous nervous system occur, which affects the function of the cardiovascular system. In the older heart "adrenergic desensitization" takes place, i.e. a decrease in the sensitivity to adrenergic stimuli, with the concomitant elevated blood catecholamines. ${ }^{20}$ This is caused by a decrease in the number of $\beta$-receptors in the $\beta$-adrenergic system and a reduced number of platelet receptors in the $\alpha$-adrenergic system with supressed reactivity to $\alpha$-adrenergic stimuli. The number of dopaminergic receptors responsible for greater contractility of the myocardium is also smaller. The ultimate effect of these changes in the autonomous nervous system in older people are weaker baroreceptor reflexes, decreased response to physiological stressors, as well as increased sensitivity to parasympathetic stimulation of the central nervous system. ${ }^{9}$

According to Ferrara, qualitative changes observed in the senile heart are similar to those in the insufficient heart. This condition, referred to as "presbycardia" or cor senile, i.e. senile heart, explains the essence of the early stage of diastolic insufficiency, which sits on the border between the physiological ageing of the heart and a pathological condition. ${ }^{20}$ The relation between systolic and diastolic cardiac failures changes with age. About $6 \%$ of patients $<60$ years of age with heart failure have diastolic insufficiency. In patients at the age of $>70$ diastolic failure makes up to $40 \%$ of the condition, whereas at the age of 80 - almost $50 \%{ }^{5}$

There are several hypotheses explaining age-related changes in the body. They include, among others, oxidation stress, inflammatory response to cellular stress, infection and apoptosis. ${ }^{9}$

Some age-related changes to the cardiovascular system may be partially reversible. Physical exercise improves endothelial function, decreases the rigidity of arteries and augments baroreceptor reflexes. With limited physical activity, older people reduce their reserves, leading to a lower threshold towards symptoms from the insufficiency of the cardiovascular system. ${ }^{9}$ The most significant similarities between the senile and insufficient hearts are presented in FIGURE and TABLE 1.

Diagnosis of CHF in older people Initial diagnosis of heart failure in the elderly may be difficult because of non-specific signs and symptoms that gradually occur. An anamnesis, which is the basis for the diagnosis of CHF, is often difficult, if not impossible, to obtain from some patients. One of the causes of failure to recognise $\mathrm{CHF}$ in the elderly is a non-specific fatigue that is attributed to ageing, whereas limited clinical manifestations may result from decreased physical activity. Symptoms of CHF such as exertional dyspnea or fatigue may be associated with other conditions such as the respiratory system diseases, metabolic diseases, anemia as well as deteriorated tolerance for physical strain. The NYHA left ventricular functional classification, based 


\begin{tabular}{l}
$\begin{array}{l}\text { Disturbance of peripheral pathomechanisms: disturbance of reflex regulation of the circulatory and respiratory } \\
\text { systems (ergo-, chemo-, baroreceptors) }\end{array}$ \\
\hline Common arrhythmias (atrial fibrillation) \\
\hline Decreased tolerance of physical exercise (decreased oxygen demand) \\
\hline Increased catabolism \\
\hline Deficiency of anabolic hormones \\
\hline Activation of inflammatory processes and coagulation (increased fibrinogen, factors V, VIII, IX etc.) \\
\hline Insulin resistance \\
\hline Nutrition disorders (lack of appetite) \\
\hline Change of the body composition (decreased muscular mass) \\
\hline
\end{tabular}

on the degree of dyspnea, is often less useful in older people. ${ }^{21-23}$ Some signs of CHF such as moist rales over the lung fields may be caused by other, non-cardiac conditions. ${ }^{23}$ An early stage of CHF may be manifested under "clinical masks" including insomnia, depression and frequent heart palpitation. ${ }^{24}$ Acute diseases (e.g. myocardial infarction, conditions with elevated temperature) or other chronic conditions such as anemia, hyper- and hypothyroidism or atrial fibrillation may aggrevate $\mathrm{CHF}$ or accelerate its appearance. Some drugs may have an unfavorable effect, e.g. non-steroidal anti-inflammatory drugs (leading to sodium retention), $\beta$-adrenolytics (with negative inotropic effects) or sympaticomimetics (increasing the afterload). ${ }^{25}$

In the diagnosis of CHF in the elderly, echocardiography plays an important role; according to the European Society of Cardiology (ESC) in 2005, it enables to determine the causes of heart failure and assessment of its type (systolic and[or] diastolic). ${ }^{26}$

Unlike middle-aged people, in whom systolic heart dysfunction is a frequent cause of heart failure, in older subjects the clinical manifestations of heart failure often occur with the correct systolic function of the left ventricle. ${ }^{27}$ Heart failure with preserved left ventricular systolic function is found in $40-80 \%$ of older people and occurs almost 2 more often in women than men. ${ }^{28,29} \mathrm{Al}$ though prognosis in patients with $\mathrm{CHF}$ with preserved left ventricular function is slightly better than for people with CHF with disturbed systolic function, the mortality risk is 4 times higher than in subjects without $\mathrm{CHF}^{30}$

The concentration of brain natriuretic peptide (BNP) or NT-proBNP recognised biochemical markers of heart failure (according to ESC), has a limited diagnostic value in older people with $\mathrm{CHF}$. The mean level of these markers rises with age. ${ }^{26,31}$ In people $>75$ years of age without heart failure, 2-3-fold higher concentration of plasma BNP was found, which may be associated with decreased glomerular filtration rate, arterial hypertension and frequent atrial fibrillation. ${ }^{25,31}$ TABLE 2 presents major differences concerning the diagnostic evaluation and treatment between patients from the younger and older age groups.
Treatment of CHF in older people Most clinical studies to date, concerning pharmacology in $\mathrm{CHF}$ (angiotensin-converting enzyme inhibitors [ACEI] and $\beta$-blockers), have been carried out in patients under 65 years of age.

The population of older persons with $\mathrm{CHF}$ considerably differs from people taking part in large clinical studies of the treatment of systolic $\mathrm{CHF}$, due to the presence of numerous additional conditions and different drug metabolisms.

While treating CHF in older people one should remember some differences which affect the therapy applied. Limited abilities to metabolise and excrete drugs should be taken into consideration. In older persons isolated diastolic heart failure is found more often, and this determines the type of therapy applied. In clinical studies performed in a population with $\mathrm{CHF}>60$ years of age, Aronow et al. ${ }^{32}$ found that the prevalence of diastolic dysfunction was $50 \%$. That is why it is important to correctly diagnose the type of dysfunction - systolic vs. diastolic - based on echocardiographic examinations, and to use the data obtained in the choice of therapy. ${ }^{33}$

Studies comprising smaller groups of older patients with $\mathrm{CHF}$ and preserved systolic left ventricular function suggest that ACEI or angiotensin receptor inhibitors may improve NYHA functional efficiency, exercise duration, ejection fraction, diastolic filling and decrease the left ventricular hypertrophy. Although calcium channel blockers are often proposed in the treatment of diastolic $\mathrm{CHF}$, there is no data from clinical studies confirming their efficacy. Nor is there data concerning the use of nitrates; however, some clinicians find them useful in reducing dyspnea if administered at bedtime. ${ }^{9}$

Older people are more at risk of adverse effects during the treatment of CHF in comparison with younger age groups. In older persons it is suggested that CHF therapy be started with small, individually selected doses, which should be gradually increased, but stopping short of the maximum ones used for younger people. ${ }^{9}$ Prior to the ad ministration of drugs it is mandatory to perform an echocardiographic examination and determine the biochemical indicators of liver and renal functions. ${ }^{34}$ 
TABLE 2 Differences between middle-aged and elderly patients with heart failure (HF)

\begin{tabular}{lll} 
Feature & Patients $<60$ years of age with HF & Patients $>70$ years of age with HF \\
$\begin{array}{l}\text { Occurrence of isolated } \\
\text { diastolic HF }\end{array}$ & $6 \%$ & $40-80 \%, 2$ times more frequently in women \\
\hline Clinical characteristics & $\begin{array}{c}\text { Occurrence of typical symptoms of HF; NYHA } \\
\text { classification helpful in diagnosis }\end{array}$ & $\begin{array}{c}\text { "Clinical masks" of HF: depression, insomnia, heart palpitation } \\
\text { Frequent complications after PTCA, CABG }\end{array}$ \\
\hline $\begin{array}{l}\text { Prognostic value of BNP } \\
\text { (NT-proBNP) }\end{array}$ & Crucial & $\begin{array}{c}\text { decreasing (2-3 } \times \text { higher concentration of BNP }>75 \text { years } \\
\text { of age in patients without HF) }\end{array}$ \\
\hline $\begin{array}{l}\text { Treatment } \\
\text { ACEl, } \beta \text {-blockers, loop diuretics, spironolactone, } \\
\text { vasodilators }\end{array}$ & $\begin{array}{l}\text { ACEl, angiotensin receptor blockers } \\
\text { Cautiously: diuretics, } \beta \text {-blockers, vasodilators }\end{array}$ \\
\hline
\end{tabular}

Abbreviations: ACEI - angiotensin-converting enzyme inhibitors, BNP - brain natriuretic peptide, CABG - coronary artery bypass graft, NT-proBNP - N-terminal pro-brain natriuretic peptide, PTCA - percutaneous transluminal coronary angioplasty

In older people, cardiac arrhythmias occur that might cause clinical manifestations of CHF. Through shortening of the diastolic phase, tachycardia decreases the duration and volume of coronary flow, resulting in lower ejection fraction. Considerable bradycardia lowers the minute cardiac volume. Dysfunction of the sinoatrial or atrioventricular nodes may lead to bradycardia and conduction disorders during $\beta$-adrenolytic therapy. Those drugs may also deteriorate ventilatory efficiency in chronic obstructive pulmonary disease or increase depression. ${ }^{9}$

Osmoreceptor defects found in the ageing process may bring about excessive dehydration during the use of loop diuretics and spironolactone. On the other hand, baroreceptor dysfunction may result in orthostatic hypotonia during the therapy with diuretics and angiotensin converting enzyme inhibitors. The administration of spironolactone-related drugs in older people may lead to the transition of occult renal failure into its clinically manifest form. That is why creatinine clearance measurement is recommended prior to therapy. After 65 years of age, glomerular filtration rate drops by $10 \%$ in 10 years, its values being 15-25\% lower in women than in men. ${ }^{35}$

In older people, especially women, higher serum levels of drugs are found in comparison with younger people receiving similar doses, which is linked to lower water content in the body. That is why saturating doses, particularly those of drugs with low therapeutic-toxic index (digoxin, lidocain, antiarrhythmics, unfractionated heparin) should be adjusted to the body weight. Intervals between doses should be longer, because in older individuals the half-life of drug elimination is longer. Hepatic clearance is lowered, hence drugs metabolised via cytochrome pathways should be administered at lower doses per time unit. Cardiac drugs whose metabolism depends on age and hepatic clearance are: $\alpha$-blockers (doxazosin), some $\beta$-blockers (metoprolol, propranolol), calcium channel blockers (dihydropyridines, verapamil, diltiazem), some HMG CoA reductase inhibitors (atorvastatin) and benzodiazepines (midazolam). ${ }^{36,37}$ The number of drugs received by older people represents another risk factor. Chronic use of 4 drugs is associated with a $50-60 \%$ risk of adverse effects, whereas the administration of 8-9 drugs increases the risk up to almost $100 \%$. A classic example of the combined effect of drugs is hypotonia and orthostatic hypotonia induced by the administration of direct vasodilators or nitrates along with $\alpha$ - or $\beta$-blockers and calcium channel inhibitors, ACEI, diuretics or sildenafil. In older persons the combination of non-steroidal anti-inflammatory drugs with ACEI may reduce potassium excretion leading to hyperkaliemia or decreased glomerular filtration rate with subsequent renal failure. ${ }^{9}$

Older people often do not comply with medical instructions, because of insufficient education, therapy costs and cognitive dysfunction. Hospitalization associated with non-compliance usually happens to solitary people. That is why it is important to involve family members or caregivers in the care of older people with heart failure.

Current 2006 ESC guidelines for pharmacological therapy of CHF in old age The problem of treating CHF in older people was discussed in the current 2005 ESC guidelines. ${ }^{26}$

Attention was focused on pharmacokinetic and pharmacodynamic differences of cardiovascular drugs in older patients, which requires special caution during therapy; lower doses are recommended. The special importance of the renal function was stressed with regard to such drugs as the majority of ACEI and digoxin which are excreted in the active form in urine. Pharmacotherapy in systolic dysfunction in older patients should be identical to that in younger persons with heart failure as far as expected outcome and drugs are concerned.

ACEI and angiotensin I receptor inhibitors These drugs are effective and well tolerated by older patients. Owing to higher risk of hypotonia and the slower excretion rate of most ACEI, it is recommended that therapy be started with small doses while monitoring potassium level and renal function parameters as well as arterial blood pressure in the supine and standing positions. ${ }^{26}$ According to the statistical data, $60 \%$ of older people are treated with ACEI of $\mathrm{AT}_{1}$-inhibitors. ${ }^{38}$ 
TABLE 3 Factors determining the variance in chronic heart failure therapy for middle-aged and elderly patients

\begin{tabular}{l}
\hline Numerous concomitant diseases \\
\hline Limited capacity for taking and eliminating drugs \\
$\begin{array}{l}\text { Increased frequency of undesirable effects related to multidrug therapy or use of specific medication (i.e. } \beta \text {-blockers-related exacerbation } \\
\text { of depression) }\end{array}$ \\
\hline $\begin{array}{l}\text { Dysfunction of sinoatrial or atrioventricular node (bradycardia and conduction disturbances can be observed during the administration } \\
\text { of } \beta \text {-adrenolytics) }\end{array}$ \\
Defect of the osmoreceptors function - excessive dehydration after the use of loop diuretics and spironolactone \\
\hline Dysfunction of baroreceptors - orthostatic hypotonia after the use of diuretics and ACEI \\
\hline Occult renal failure - administration of spironolactone may lead to renal failure \\
\hline Among the elderly, decreased hepatic clearance of specific medication: $\alpha$-blockers (doxazosin), some $\beta$-blockers (metoprolol, propranolol), \\
calcium channel blockers (dihydropyridines, verapamil, diltiazem), some HMG-CoA reductase inhibitors (atorvastatin) and benzodiazepine \\
derivatives (midazolam). At high doses these medications may be toxic - dose correction on the basis of body mass is necessary \\
\hline More frequent occurrence of diastolic heart failure \\
\hline Increased frequency of complications after PCI and CABG procedures \\
\hline Noncompliance
\end{tabular}

Abbreviations: $\mathrm{PCl}$ - percutaneous coronary intervention, others - see TABLE 2

Diuretics Diuretics are taken by about $90 \%$ of older patients with $\mathrm{CHF}^{38}$ In older patients, thiazides are often ineffective due to decreased glomerular filtration rate. Lower absorption and bioavailability of drugs and higher level of the excretion of thiazides and loop diuretics may lead to delayed therapeutic effect and their prolonged or sometimes lower activity. On the other hand, diuretics often cause orthostatic hypotonia and(or) further deterioration of renal function. In older patients hyperkaliemia is more often observed with the use of combined aldosterone antagonists and ACEI or non-steroidal anti-inflammatory drugs and coxibes. ${ }^{26}$

$\beta$-blockers $\beta$-blockers are well tolerated by older patients unless there are contraindications such as conduction disorders, bradycardia or pulmonary diseases. Therapy with $\beta$-blockers in older patients should be started with low doses, which should be increased at longer intervals. ${ }^{26} \beta$-blockers that are currently used for heart failure are excreted by the liver and do not require dose reduction in patients with impaired renal function.

A meta-analysis of more than 12,000 patients participating in large clinical studies with $\beta$-blockers (BEST, CARVEDILOL-US, CIBIS III, COPERNICUS, MERIT-HF) showed that they considerably reduced the mortality rate in older patients as well. ${ }^{39}$ In spite of this, the data from EuroHeart Failure Survey Programme showed that only about $25 \%$ of older patients with $\mathrm{CHF}$ are treated with $\beta$-blockers. ${ }^{38}$

When discussing $\beta$-blockers, a recently published clinical study called SENIORS should be mentioned, in which the effect of nebivolol was evaluated on the reduction of the mortality risk for any reason or hospitalization for cardiovascular causes in patients with $\mathrm{CHF}>70$ years of age. In the study, the use of nebivolol (a selective $\beta_{1}$-blocker) was found to be associated with a $16 \%$ reduction in the risk of death for cardiovascular reasons. Analyzing the data obtained from the subgroup of patients of $<75$ years of age with LVEF $\leq 35 \%$ and comparing them with the results of other studies, the authors concluded that the efficacy of nebivolol was similar to that of metoprolol, bisoprolol and carvedilol in patients with $\mathrm{CHF}^{40}$

Cardiac glycosides According to the ESC guidelines, cardiac glycosides are indicated in patients with atrial fibrillation in order to control the ventricular rate. In sinus rhythm, they are indicated in patients with severe systolic left ventricular dysfunction receiving ACEI, $\beta$-blockers, diuretics and spironolactone. Older patients are more vulnerable to adverse effects of digoxin. It is predominantly excreted by the kidneys in the active form, so its half-life may be prolonged 2- or 3-fold in patients of $>70$ years of age. That is why doses between 0.0625 and $0.125 \mathrm{mg} / 24 \mathrm{~h}$ are recommended for older patients. In patients with elevated creatinine levels digoxin doses should be adjusted accordingly. ${ }^{26}$

Vasodilators Drugs from this group (nitrates, dihydralazine) and combinations of them should be used with great caution due to a high risk of hypotonia. There is scarce data concerning the efficacy and safety of these medications in treating heart failure in older patients. ${ }^{26}$

Non-pharmacological strategies in older patients In patients with $\mathrm{CHF}$ reduction of salt intake and moderate exercise are recommended. Resynchronisation therapy may reduce the frequency of hospitalisation and mortality in selected patients with symptomatic systolic heart failure and prolonged repolarization or QRS in ECG. ${ }^{41}$

Ischemic etiology of heart failure requires the consideration of invasive therapy in some patients. Experience concerning revasculariztion procedures such as percutaneous transluminal 
coronary angioplasty (PTCA) and coronary artery bypass grafting (CABG) in older people is growing. Half of these procedures are performed in patients of $>65$ years of age, and $1 / 3$ of coronary revascularization procedures are performed in persons of $>70$ years of age. Randomized studies show the efficacy and positive outcome of these therapeutic modalities, though in a limited number of older patients. ${ }^{9}$ The greatest number of patients of $>75$ years of age (109 persons) with a multi-vessel disease were recruited by the BARI. ${ }^{42}$ Among persons of $65-80$ years of age early morbidity and mortality rates after CABG were higher than those following PTCA, but $C A B G$ was associated with resolution of angina and rarer repeat interventions. ${ }^{42}$

In the TIME trial, invasive therapy (PTCA or CABG) was compared with optimum pharmacological treatment in patients with CAD (coronary artery disease) at the age of $>75$, with angina pectoris refractory to standard therapy. Although initial analysis after 6 months showed the benefits of revascularization, after 1 year these benefits were no longer visible. Revascularization was associated with a higher risk of early death and complications, whereas optimal pharmacology implied higher risk of incidents during follow-up (requiring hospitalization and revascularization) with no clear advantage of any strategy. ${ }^{43}$

With age, invasive procedures are accompanied by more frequent complications. PTCA in older patients is associated with less than $1 \%$ risk of persistent stroke or coma, whereas $C A B G$ - with 3-6\% risk of persistent stroke or coma in persons of $>75$ years of age. Immediately after the procedure a longer period of artificial ventilation is observed, inotropic drugs of intraaortic contrapulsation are more often required and complications such as bleeding, renal insufficiency, periprocedural myocardial infarction and infections are more frequent. The highest complication rate is usually found in older women and in patients undergoing emergency procedures. Apart from a higher early mortality and morbidity rate associated with revascularization in older patients the period of disability and rehabilitation following procedures in older people is usually longer. ${ }^{9}$

Complications occurring during invasive procedures, which are uncommon among younger patients, reflect the biological status and the coexistence of other conditions in older patients. Decisions concerning the strategy of therapy for CAD (pharmacology, PTCA or CABG) should be based on the potential effect of the disease on the patient's biological status, lifestyle and individual preferences.

There are several essential factors determining the differences of therapy between patients in middle and older ages (TABLE 3).

The above-presented data from the literature shows that the treatment of heart failure in older persons is a great challenge that should take into consideration a number of health problems resulting from the ageing of the body.

\section{REFERENCES}

1 Lloyd-Jones DM. The risk of congestive heart failure: sobering lessons from the Framingham Heart Study. Curr Cardiol Rep. 2001; 3: 184-190.

2 Davies M, Hobbs F, Davis R, et al. Prevalence of left-ventricular systolic dysfunction and heart failure in the Echocardiographic Heart of England Screening study: a population-based study. Lancet. 2001; 358: 439-444

3 Gottdiener JS, Arnold AM, Aurigemma GP, et al. Predictors of congestive heart failure in the elderly: the Cardiovascular Health Study. J Am Coll Cardiol. 2000; 35: 1628-1637.

4 Wang TJ, Levy D, Benjamin EJ, Vasan RS. The epidemiology of "asym ptomatic" left ventricular systolic dysfunction: implications for screening. Ann Intern Med. 2003; 138: 907-916.

5 Alexander KP, Peterson ED. [Approach to the Elderly Patient with Heart Disease]. In: Braunwald E, Goldman L. [Primary Cardiology]. Urban \& Partner, Wrocław 2005; 259-269. Polish.

6 Croft JB, Giles WH, Pollard RA, et al. Heart failure survival among older adults in the United States: a poor prognosis for an emerging epidemic in the Medicare population. Arch Intern Med. 1999; 159: 2225-2226.

7 Pilote L, Dasgu pta K, Guru V et al. A comprehensive view of sex-specific issues related to cardiovascular disease. CMAJ. 2007; 176: S1 $-S 44$.

8 Gustafsson F, Torp-Pedersen C, Burchardt $\mathrm{H}$, et al. Female sex is associated with a better long-term survival in patients hospitalized with congestive heart failure. Eur Heart J. 2004; 25: 129-135.

9 Schwarz JB, Zipes Douglas P. Cardiovascular disease in the elderly In: Zipes Douglas $P$, Braunwald $E$, eds. Braunwald's heart disease: a textbook of cardiovascular medicine. Philadelphia, W.B. Saunders, 2005; 1925-1949.

10 Lakatta EG, Levy D. Arterial and cardiac aging: major shareholders in cardiovascular disease enterprises: Part I: aging arteries: a "set up" fo vascular disease. Circulation. 2003; 107: 139-146.

11 Kass DA. Age-related changes in venticular-arterial coupling: pathophysiologic implications. Heart Fail Rev. 2002; 7: 51-62.

12 Lakatta EG. Arterial and cardiac aging: major shareholders in cardiovascular disease enterprises: Part III: cellular and molecular clues to heart and arterial aging. Circulation. 2003; 107: 490-497.

13 Besse S, Delcayre C, Chevalier B, et al. Is the senescent heart overloaded and already failing? Cardiovasc Drugs Ther. 1994; 8: 581-587.

14 Lakatta EG. Aging and cardiovascular structure and function in healthy sedentary humans. Aging (Milano). 1998; 10: 162-164.

15 Marchionni N, Di Bari M, Innocenti F, et al. Age and left ventricular function. Aging (Milano). 1998; 10: 165-166.

16 Little WC, Zile MR, Kitzman DW, et al. The effect of alagebrium chloride (ALT-711), a novel glucose cross-link breaker, in the treatment of elderly patients with diastolic heart failure. J Card Fail. 2005; 11: 191-195.

17 Bakris GL, Bank AJ, Kass DA, et al. Advanced glycation end-product cross-link breakers. A novel approach to cardiovascular pathologies related to the aging process. Am J Hypertens. 2004; 17 (12 Pt 2): S23 -S30.

18 Lakatta EG, Sollott SJ. Perspectives on mammalian cardiovascular ag ing: humans to molecules. Comp Biochem Physiol A Mol Integr Physiol. 2002; 132: 699-721.

19 Willerson JT. Systemic and local inflammation in patients with unstable atherosclerotic plaques. Prog Cardiovasc Dis. 2002; 44: 469-478.

20 Ferrara N, Davia K, Abete P, et al. Alterations in beta-adrenoceptor mechanisms in the aging heart. Relationship with heart failure. Aging (Milano). 1997; 9: 391-403.

21 Gąsowski J, Grodzicki T. [Chronic circulatory insufficiency in the elderly]. Gerontol Pol. 1996; 4: 41-50. Polish.

22 Pędich W. [Chronic stasis cardiac insufficiency: geriatric aspects] Nowa Med. 1996; 3: 9-11. Polish.

23 Żakowska-Wachełko B, Pędich W. [Elderly Patients]. PZWL, Warszawa 1995. Polish.

24 Pędich W, Szreniawski Z. [Treatment of chronic stasis circulatory insufficiency]. In: Pędich W, Szreniawski Z, eds. [Geriatric Pharmacotherapy] Warszawa, PZWL, 1998; 90-108. Polish.

25 Grodzicki T, Fedyk-tukasik M. [Cardiac insufficiency in the elderly]. In: Dubiel JS, Korewicki J, Grodzicki T, eds. [Cardiac Insufficiency]. Gdańsk, Via Medica, 2004; 153-161. Polish.

26 Swedberg K, Cleland J, Dargie H, et al. Guidelines for the diagnosis and treatment of chronic heart failure: executive summary (update 2005) The Task Force for the Diagnosis and Treatment of Chronic Heart Failure of the European Society of Cardiology. Eur Heart J. 2005; 26: 1115-1140.

27 Wendelboe Nielsen 0 , Kirk V, Bay M, et al. Value of N-terminal pro brain natriuretic peptide in the elderly: data from the prospective $\mathrm{Co}_{0}$ penhagen Hospital Heart Failure study (CHHF). Eur J Heart Fail. 2004; 6 $275-279$ 
28 Aronow WS, Ahn C, Kronzon I. Normal left ventricular ejection fraction in older persons with congestive heart failure. Chest. 1998; 113: 867-869.

29 Chodorowski Z. [Stasis cardiac insufficiency in the elderly]. Post Nauk Med. 1999; 12: 15-22. Polish.

30 Kitzman DW, Gardin JM, Gottdiener JS, et al. Importance of heart failure with preserved systolic function in patients $\geq 65$ years of age. CHS Research Group. Cardiovascular Health Study. Am J Cardiol. 2001; 87 413-419.

31 Masoudi FA, Havranek EP, Smith G, et al. Gender, age, and heart failure with preserved left ventricular systolic function. J Am Coll Cardiol. 2003; 41: 217-223.

32 Philbin EF, Erb T, Jenkins P. The natural history of heart failure with preserved left ventricular systolic function. J Am Coll Cardiol. 1997; 29 (2 Suppl A): A245

33 Redfield MM, Jacobsen SJ, Burnett JC Jr, et al. Burden of systolic and diastolic ventricular dysfunction in the community: appreciating the scope of the heart failure epidemic. JAMA. 2003; 289: 194-202.

34 Chodorowski Z. [Chronic stasis cardiac insufficiency in the elderly] Nowa Klin. 2000; 7: 836-844. Polish.

35 Levey AS, Bosch JP, Lewis JB, et al. A more accurate method to estimate glomerular filtration rate from serum creatinine: a new prediction equation. Modification of Diet in Renal Disease Study Group. Ann Intern Med. 1999; 130: 461-470.

36 Kang D, Verotta D, Krecic-Shepard ME, et al. Population analyses of sustained-release verapamil in patients: effects of sex, race, and smoking. Clin Pharmacol Ther. 2003; 73: 31-40.

37 Schwartz JB. Gender-specific implications for cardiovascular medication use in the elderly optimizing therapy for older women. Cardiol Rev. 2003; 11: 275-298.

38 Cleland JG, Swedberg K, Follath F, et al. The EuroHeart Failure survey programme - a survey on the quality of care among patients with heart failure in Europe. Part 1: patient characteristics and diagnosis. Eur Heart J. 2003; 24: 442-463.

39 Dulin BR, Haas SJ, Abraham WT, Krum H. Do elderly systolic heart failure patients benefit from beta blockers to the same extent as the non-elderly? Meta-analysis of $>12,000$ patients in large-scale clinical trials. Am J Cardiol. 2005; 95: 896-898.

40 Flather MD, Shibata MC, Coats AJ, et al. Randomized trial to determine the effect of nebivolol on mortality and cardiovascular hospital admission in elderly patients with heart failure (SENIORS). Eur Heart J. 2005 26: $215-225$.

41 Bradley DJ, Bradley EA, Baughman KL, et al. Cardiac resynchronization and death from progressive heart failure: a meta-analysis of randomized controlled trials. JAMA. 2003; 289: 730-740.

42 Mullany CJ, Mock MB, Brooks MM, et al. Effect of age in the Bypass Angioplasty Revascularization Investigation (BARI) randomized trial. Ann Thorac Surg. 1999; 67: 396-403.

43 TIME Investigators. Trial of Invasive versus Medical therapy in Elderly patients with chronic symptomatic coronary-artery disease (TIME): a randomised trial. Lancet. 2001; 358: 951-957. 